\title{
The Nature of Metal-Metal Bonding in Re- and Os-Corrole Dimer
}

\author{
Mohammed Obies*a and Aqeel A. Hussein*b
}

College of Pharmacy, University of Babylon, 51002, Hillah/Babylon, Iraq; E-mail: phar.mohammed.h.obies@uobabylon.edu.iq

Department of Pharmacy, College of Medicine, Komar University for Science and Technology, Qularaisi, Sulaymaniyah, Kurdistan Region, Iraq; Email: aqeel.alaa@komar.edu.iq

Abstract: Studies of multiple bonding between transition metals complexes offer fundamental insight into the nature of bonding between metal ions and facilitate predictions of the physical properties and the reactivities of metal complexes containing metal-metal multiple bonds. Here we report a computational interrogation on the nature of the metal-metal bonding for neutral, oxidized, and reduced forms of dinuclear rhenium and osmium corrole complexes, $\left[\{\operatorname{Re}[\mathrm{TpXPC}]\}_{2}\right]^{0 / 1+1-}$ and $\left[\{\mathrm{O}[\mathrm{TpXPC}]\}_{2}\right]^{0 / 1+11-}$, using complete active space self-consistent (CASSCF) methodology and density functional theory (DFT) calculations. For $\left[\{\operatorname{Re}[T p X P C]\}_{2}\right]^{0}$ and $\left[\{O s[T p X P C]\}_{2}\right]^{0}$, CASSCF calculations shows that the effective bond order is 3.29 and 2.73 , respectively. On their oxidized forms, $\left[\{\operatorname{Re}[\mathrm{TpXPC}]\}_{2}\right]^{1+}$ and $\left[\{\mathrm{OS}[\mathrm{TpXPC}]\}_{2}\right]^{1+}$ molecules, the results indicate an electron removal from a ligand-based orbital, where $\left[\{\operatorname{Re}[T p X P C]\}_{2}\right]^{1+}$ gives slightly different geometry from its neutral form due to populating the $\delta^{*}$ orbital. In this regard, the CASSCF calculations give an effective bond order of 3.25 which is slightly lower than in the $\left[\{\operatorname{Re}[T p X P C]\}_{2}\right]^{0}$. On their reduced forms, the electron addition appears to be in the metal-based orbital for $\left[\{\operatorname{Re}[T p X P C]\}_{2}\right]^{1-}$ whereas in the ligand-based orbital for the Os-analogue which has no effect on the Os-Os bonding, presenting an effective bond order of 3.18 for the $\left[\{\operatorname{Re}[T p X P C]\}_{2}\right]^{1-}$ within the CASSCF simulations. These results will further encourage theoreticians and experimentalists to design metalloporphyrin dimers with distinct metal-metal bonding.

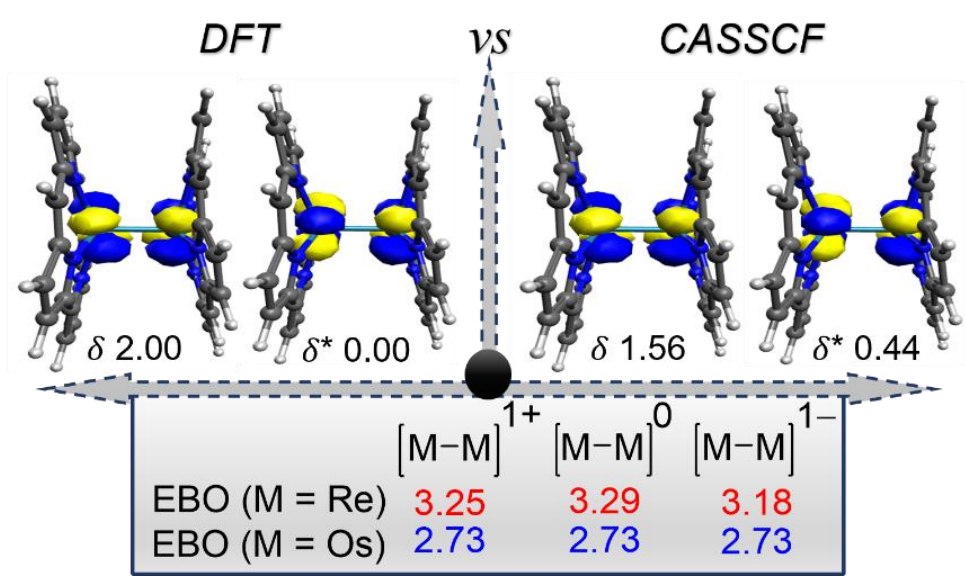




\section{Introduction}

Corrole ${ }^{1}$ is a contracted porphyrin analogue that can be bound to transition metals giving so-called metallocorroles. It is known to stabilize transition metals in high oxidation states. ${ }^{2}$ Metallocorroles are useful in various catalytic reactions, for example, iron and manganese corroles proved to be useful in hydroxylation reactions whereas antimony and rhodium corroles were successfully used in oxidation reactions of hydrocarbons and cycloporpanation reactions, respectively. Furthermore, iron and rhodium corroles have been also used in $\mathrm{N}-\mathrm{H}$ and $\mathrm{C}-\mathrm{H}$ insertion reaction, respectively. ${ }^{3}$ All these molecules have single transition metal in their structures. However, metalloporphyrin also can be synthesis as dimers with multiple metal-metal bonds in which have a significant interest. ${ }^{4}$ Tuning the bond order of the metalloporphyrin/corrole dimers can be achieved by oxidation and reduction of the dimer. Metal-metalbonded dimeric porphyrin complexes in various accessible oxidation states were studied utilizing resonance Raman scattering, infrared absorption, and absorption spectroscopy. Error! Bookmark not defined.d,e For instance, Kadish and co-workers ${ }^{5}$ characterized the one-electron-oxidized forms of ruthenium-rutheniumbonded dimeric corrole complexes using combination of UV/Vis spectrophotometry and EPR Spectroelectrochemistry. Collman and Arnold conducted experimental (UV/Vis/NIR/EPR spectroelectrochemical) and theoretical (DFT) approaches to establish the electronic nature of the electrogenerated species. ${ }^{4}$

Interestingly, transition metal corrole dimer $\{\mathrm{M}[\mathrm{TPXPC}]\}_{2}(\mathrm{M}=\mathrm{Re}$ or Os and TpXPC refers to a mesotris(para-X-phenyl) corrole and $\mathrm{X}=\mathrm{CF}_{3}, \mathrm{H}, \mathrm{Me}$, and $\mathrm{OMe}$ ) have been synthesized recently by Ghosh and coworkers. ${ }^{6}$ The cyclic voltammetry measurements of the $\{\operatorname{Re}[\mathrm{TpMePC}]\}_{2}$ and the $\{\mathrm{Os}[\mathrm{TpMePC}]\}_{2}$ exhibit three reversible oxidations and one reversible reduction. The X-ray data is available just for the neutral structure. Thus, there is little information regarding the nature of the metal-metal bonding in the oxidized and reduced species from electrochemistry and DFT calculations. ${ }^{6 a, b}$ Hight level computational calculations offer fundamental insight into the nature of metal-metal bonding in the neutral, oxidized, and reduced species of the $\left[\{\operatorname{Re}[\mathrm{TpMePC}]\}_{2}\right]^{0 / 1+/ 1-}$ and the $\left[\{\mathrm{O}[\mathrm{TPMePC}]\}_{2}\right]^{0 / 1+/ 1-}$ complexes. However, rationalizing the nature of metal-metal bonding is challengeable from theoretical perspectives. ${ }^{7}$

The most popular computational method is DFT. It has been used for dealing with large systems, in particular, those that contain transition metals. This method, however, in some cases fails to give a clear description of the nature of the metal-metal bonding because it is inherently a single determinant in nature, and the accuracy of the available exchange-correlation functionals is still open to debate. Metalmetal bonding forms from the overlap between d orbitals on each metal of the dimer. This leads to forming 
different types of bonds that are $\sigma, \pi$, and $\delta$. These bond components have different strengths; therefore, they cannot be described by DFT because it does not take into account the very different contributions of the $\sigma, \pi$, and $\delta$ components to the overall bond strength. An alternative approach is multi-configurational self-consistent field (MCSCF) method, specifically, the complete active space self-consistent field (CASSCF) method. This approach takes into account the different contributions of the $\sigma, \pi$, and $\delta$ components of overall bond strength, thus, gives a better description of the nature of the metal-metal bonding. The main challenge in using the CASSCF methodology is the choice of the active space due to its dependence on the chemical intuition of the system under study. The occupation numbers in single determinant methods (DFT) are restricted to integer values whereas in the CASSCF wavefunction can be fractions between zero and two which can capture all points between the strongly bonded limit and the dissociation limit, where occupation numbers of the bonding and antibonding orbitals are 0.5.

(a)

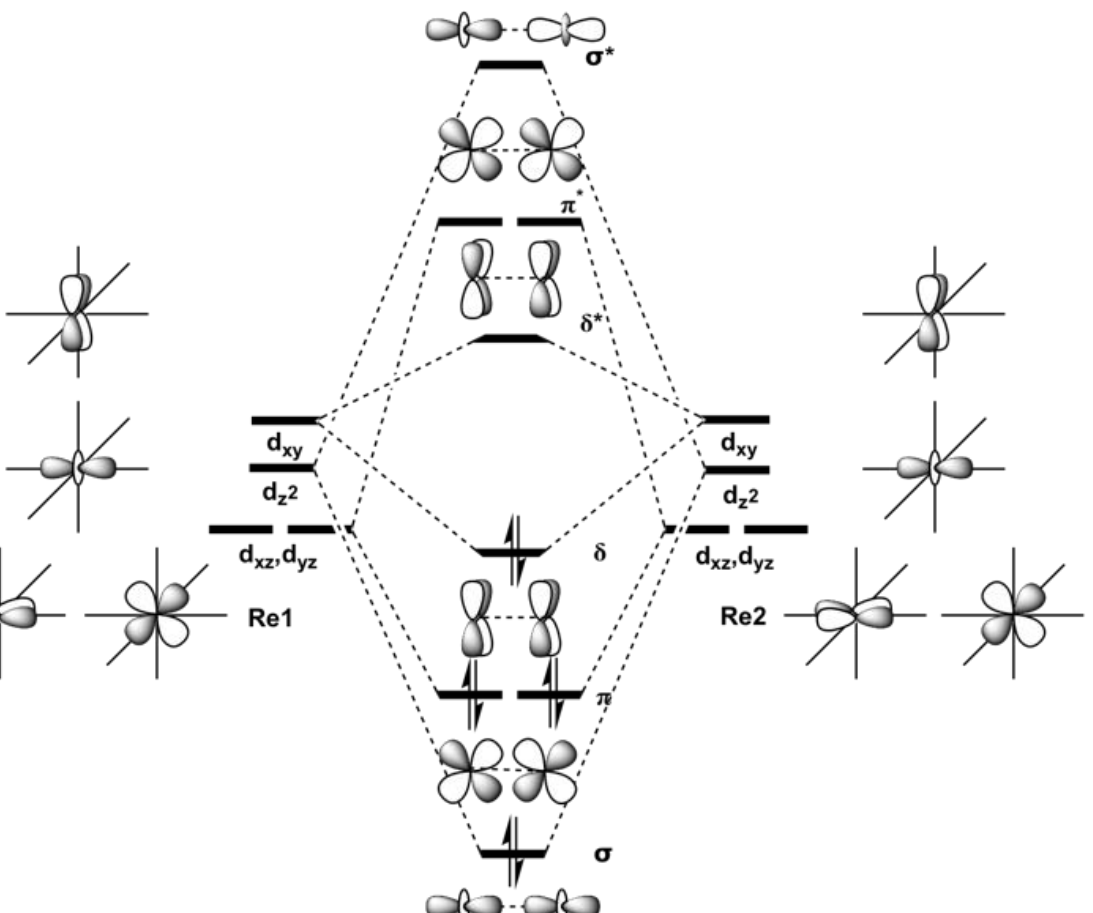

(b)
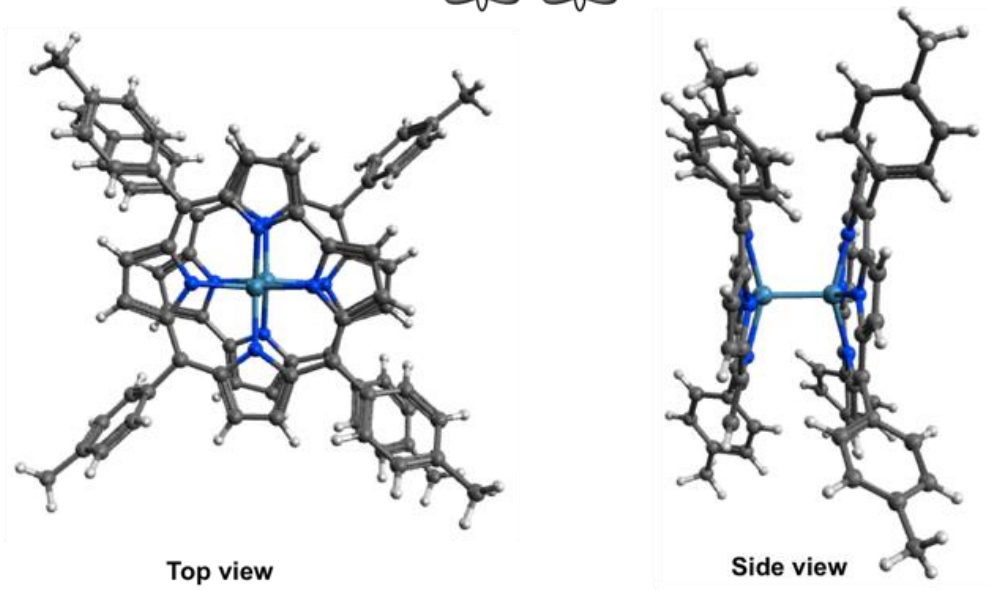
Fig. 1. (a) Schematic molecular orbital arrays for $D_{4 h}-\left[\operatorname{Re}_{2} \mathrm{Cl}_{8}\right]^{2-}$ molecule and (b) $X$-ray structure of $\{M[T p M e P C]\}_{2}$ ( $\mathrm{M}=\mathrm{Re}$ or Os) complex with two views.

The $\left[\{\operatorname{Re}[\mathrm{TpXPC}]\}_{2}\right]^{0}$ and $\left[\{\mathrm{OS}[\mathrm{TpXPC}]\}_{2}\right]^{0}$ complexes have similar geometries to the landmark $\left[\operatorname{Re}_{2} \mathrm{Cl}_{8}\right]^{2-}$ molecule. ${ }^{8}$ Each metal centre of the dimers is bonded to the ligands through four nitrogen atoms. The nitrogen atoms have almost an eclipsed arrangement similar to the chlorine atoms in the $\left[\operatorname{Re}_{2} \mathrm{Cl}_{8}\right]^{2-}$. Therefore, the metal-metal bonding of the $\left[\{\operatorname{Re}[\mathrm{TpXPC}]\}_{2}\right]^{0}$ and $\left[\{\mathrm{OS}[\mathrm{TpXPC}]\}_{2}\right]^{0}$ complexes can be described similarly to that in the $\left[\mathrm{Re}_{2} \mathrm{Cl}_{8}\right]^{2-}$ molecule. The $\left[\mathrm{Re}_{2} \mathrm{Cl}_{8}\right]^{2-}$ molecule features a metal-metal quadruple bond with a Re-Re distance of only $2.27 \AA .^{8}$ The structure of this molecule has been described as two rhenium atoms lying within a square prism defined by eight chlorine atoms. The bonding between the two rhenium centres was explained qualitatively using molecular orbital arrays as shown in Fig. 1a. The four $d$ orbitals $\left(d_{z^{2}}, d_{x z}, d_{y z}\right.$, and $\left.d_{x y}\right)$ on each metal centers are participated in the Re-Re bonding $(\sigma, \pi$, and $\delta)$ while the $d_{x^{2}-y^{2}}$ orbitals on each rhenium atom interact with the ligands. $\operatorname{Re}_{2}$-core has eight electrons populating the four bonding orbitals in Fig. 1a $\left(\sigma^{2} \pi^{4} \delta^{2}\right)$ giving a bond order of 4.0. The metal-metal bonding of $\left[\mathrm{Mo}_{2} \mathrm{Cl}_{8}\right]^{4-},\left[\mathrm{TC}_{2} \mathrm{Cl}_{8}\right]^{2-}$, and $\left[\mathrm{Tc}_{2} \mathrm{Cl}_{8}\right]^{3-}$ complexes is described in the same way as that in the $\left[\mathrm{Re}_{2} \mathrm{Cl}_{8}\right]^{2-} .{ }^{9}$

In 2003, Gagliardi and Roos studied the electronic structure of $\left[\mathrm{Re}_{2} \mathrm{Cl}_{8}\right]^{2-}$ using CASSCF/PT2 methodology. ${ }^{10 a}$ Them calculations adopted a $\operatorname{CAS}(12,12)$ active space that includes the eight metal-based orbitals (Fig. 1a) and the four metal-ligand orbitals and twelve electrons distributed among them. The CASSCF results showed that the ground state occupations of the $\delta$ and $\delta^{*}$ orbitals are 1.54 and 0.46 , respectively. These occupation numbers are very different from the classical values of $2.0(\delta)$ and $0.0\left(\delta^{*}\right)$ for formally doubly occupied and unoccupied molecular orbitals. For the $\sigma$-manifold the natural orbital populations are 1.92 and 0.08 for $\sigma$ and $\sigma^{*}$, respectively, reflecting substantial overlap of the $\sigma$ orbitals. The natural orbital populations of $\pi$-symmetry are intermediate, with values of 3.74 and 0.26 for bonding and antibonding orbitals, respectively. These occupations give a total Re-Re bond order of 3.18, much smaller than the classical value of 4.0. The metal-ligand orbitals remain almost fully occupied in the wavefunction with bonding and antibonding occupations of 1.98 and 0.02 , respectively. The effective bond order of the $\left[\mathrm{Re}_{2} \mathrm{Cl}_{8}\right]^{2-}$ molecule was also calculated by Sakaki and co-workers using a CAS(8,8), the metal-ligand orbitals were not included in the calculations. ${ }^{10 \mathrm{~b}}$ The results show that the effective Re-Re bond order of 3.20 is very close to the Roos and co-workers results.

Therefore, we believe that understanding the nature of the metal-metal bonding in the transition metal corrole dimers is still in need of further investigation using computational methods that are beyond the single determinant method (DFT). Here we report a computational study on the $\{\mathrm{M}[\mathrm{TpMePC}]\}_{2}(\mathrm{M}=\operatorname{Re}$ or Os) complexes and their oxidation and reduction forms to shed some light on the nature of the metal-metal 
bonding in these important complexes using the complete active space self-consistent field (CASSCF) methodology.

\section{Results and Discussions}

Initial considerations. In this computational study, all geometries including neutral, oxidized, and reduced species were optimized using two different exchange-correlation functionals that are OLYP and B3LYP. The atom-pairwise dispersion correction with the Becke-Johnson damping scheme (D3BJ) was applied on all optimized geometries. To reduce the computational cost, a model of the TpMePC ligand where used. The phenyl rings of the ligands with their substitutions are replaced by hydrogen atoms. To validate this simplification on the structure parameters, we have firstly compared the main structure parameters of the complex with the full ligand and with the model ligand. Following this validation, we will then present a detailed analysis of the neutral, oxidized, and reduced species using the simplified model of the ligand TpMePC. In the following discussion, we will refer to the simplified complex as $\mathrm{M}_{2}-$ Model and $\mathrm{M}_{2}-\mathrm{Full}$ ( $\mathrm{M}=$ Re or Os) for the complex with the whole ligand.

Geometrical comparison: Table 1 illustrates the main geometry parameters of the $\left[\mathrm{M}_{2}-\mathrm{Model}\right]^{0}$ and the $\left[\mathrm{M}_{2}-\mathrm{Full}\right]^{0}$ complexes computed with OLYP functional along with the crystallographic data. The Re-Re bond length in the $\left[\mathrm{Re}_{2}-\right.$ Model] ${ }^{0}$ molecule is computed to be $2.20 \AA$ compared to $2.2364(6) \AA$ of its X-ray counterpart whereas $2.19 \AA$ with the full ligand. The averaged Re-N bond distance of the model ligand is reproduced within $0.01 \AA$ of crystallographic value and within $0.02 \AA$ with the $\left[\mathrm{Re}_{2}-\mathrm{Full}\right]^{0}$. The Os-Os bond distance is also in good agreement with the $X$-ray values since the computed value is shorter than the $X$ ray value by only $0.02 \AA$ of the $\left[\mathrm{Os}_{2}-\mathrm{Model}\right]^{0}$ and the $\left[\mathrm{Os}_{2}-\mathrm{Full}\right]^{0}$. The averaged Os- $\mathrm{N}$ distance of both $\left[\mathrm{Os}_{2^{-}}\right.$ Model $]^{0}$ and $\left[\mathrm{Os}_{2}-\mathrm{Full}\right]^{0}$ is predicted within $0.02 \AA$ compared to the X-ray result. These results show that the simplification has almost no effect on the structure parameters, thus, the model structure will be used in all electronic structure analysis in this paper. Also, these results indicate that the computational methodology is valid for electronic structure analysis of the $\{\operatorname{Re}[\mathrm{TpMePC}]\}_{2}{ }^{0}$ and $\{\mathrm{OS}[\mathrm{TpMePC}]\}_{2}{ }^{0}$ molecules, and consequently for the oxidation and reduction forms.

Table 1. Comparison of the optimized structural parameters using OLYP of $\{\mathrm{M}[\mathrm{TpMePC}]\}_{2}{ }^{0}(\mathrm{M}=\mathrm{Re}$ or Os) with its model ligands and crystallographic data. The Os-complex values are in parentheses. Distances are in $\AA$ and Avg. stand for average.

$\mathrm{M}=$ Re or $\mathrm{M}_{2}$-Model $\quad \mathrm{M}_{2}$-Full $\quad$ X-ray
Os




\begin{tabular}{lccc}
\hline M-M & $2.20(2.22)$ & 2.19 & $2.2364(6)$ \\
& & $(2.22)$ & $(2.2403(5))$ \\
Avg. M-N & $2.02(1.98)$ & 2.03 & $2.01(1.96)$ \\
& & $(1.98)$ & \\
\hline
\end{tabular}

$\left[\operatorname{Re}_{2}-\text { Model }\right]^{0}$ and $\left[\mathrm{Os}_{2}-\text { Model] }\right]^{0}:$ Kohn-Sham molecular orbital array of the $\left[\operatorname{Re}_{2}-\text { Model }\right]^{0}$ complex is shown in Fig. 2. The eight electrons of the $\mathrm{Re}_{2}$-core populate the bonding orbitals in Fig. 2 giving a low spin ground state with $\sigma^{2} \pi^{4} \delta^{2}$ electron configuration and formal bond order of 4.0. The Os ${ }_{2}$-core in the [Os ${ }_{2}$-Model] ${ }^{0}$ molecule has ten electrons which are populating the $\delta^{*}$ orbital beside the $\sigma, \pi$, and $\delta$ orbitals. That gives a $\sigma^{2} \pi^{4} \delta^{2} \delta^{* 2}$ electron configuration with a bond order of 3.0. Despite the fact that the bond order of [ $\left[\mathrm{Re}_{2^{-}}\right.$ Model $]^{0}$ and $\left[\mathrm{Os}_{2}-\right.$ Model] ${ }^{0}$ complexes are different (4.0 vs 3.0), the metal-metal bond distance in both $\{\operatorname{Re}[\mathrm{TpMePC}]\}_{2}{ }^{0}$ and $\{\mathrm{Os}[\mathrm{TpMePC}]\}_{2}{ }^{0}$ systems is the same ( 2.24 $\AA$ ). However, our DFT-OLYP calculations predict the metal-metal bond in the $\left[\mathrm{Os}_{2}-\mathrm{Model}^{0}\right.$ molecule slightly longer than in the Re-analogue (the difference is $0.02 \AA$ ). That could be attributed to the accuracy of exchange-correlation functional. DFT with B3LYP functional predicted the M-M bond length of $2.22 \AA$ in the $\left[\operatorname{Re}_{2}-\text { Model }\right]^{0}$ complex and $2.23 \AA$ in the $\left[\mathrm{Os}_{2}-\mathrm{Model}\right]^{0}$ molecule, showing that the M-M bond length depends on the applied exchange-correlation functional (the difference between $\mathrm{M}-\mathrm{M}$ bond length of the $\left[\mathrm{Re}_{2}-\mathrm{Model}\right]^{0}$ and the $\left[\mathrm{Os}_{2}-\mathrm{Model}\right]^{0}$ of $0.01 \AA$ compared to $0.02 \AA$ with OLYP results). The similarity between the bond length of both systems can be explained with similar considerations to that of $\left[\mathrm{Tc}_{2} \mathrm{Cl}_{8}\right]^{2-/ 3-}$ complex even though the Tc-Tc bond length of these systems is different as we will see shortly. ${ }^{10}$ The formal bond order of the $\left[\mathrm{Tc}_{2} \mathrm{Cl}_{8}\right]^{2-}$ systems is equal to $4.0\left(\sigma^{2} \pi^{4} \delta^{2}\right)$ and for the $\left[\mathrm{Tc}_{2} \mathrm{Cl}_{8}\right]^{3-}$ equal to $3.50\left(\sigma^{2} \pi^{4} \delta^{2} \delta^{* 1}\right)$. The Tc-Tc bond distance should be shorter in the $\left[\mathrm{Tc}_{2} \mathrm{Cl}_{8}\right]^{2-}$ systems compare to that in the $\left[\mathrm{Tc}_{2} \mathrm{Cl}_{8}\right]^{3-}$. However, the $\mathrm{X}$-ray data and so the quantum chemical calculations show a reverse trend (the computed Tc-Tc bond length for the $\left[\mathrm{Tc}_{2} \mathrm{Cl}_{8}\right]^{3-}$ system is

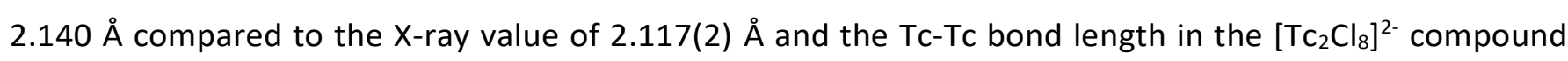
calculated of $2.170 \AA$ compared to 2.1560 (3) $\AA$ of the X-ray). This trend is attributed to many reasons: crystal packing, the $\pi$ component of the Tc-Tc bond is larger in the $\left[\mathrm{Tc}_{2} \mathrm{Cl}_{8}\right]^{3-}$, and maybe the $\delta$ bond order is lower in the $\left[\mathrm{Tc}_{2} \mathrm{X}_{8}\right]^{3-}$ because the single occupation of the $\delta^{*}$ orbital which explains the small internal rotation in the $\left[\mathrm{TC}_{2} \mathrm{Cl}_{8}\right]^{3-}$ compound.

Based on the aforementioned results, the single determinant description from DFT on the metal-metal bonding does not take into account the different strengths of the M-M bonding components $(\sigma, \pi$, and $\delta$ ). Therefore, we have turned our attention to the multi-configurational method, namely CASSCF. For the $\left[\mathrm{Re}_{2}-\mathrm{Model}\right]^{0}$ system, the active space consists of the metal-based orbitals that are $\sigma, 2 \pi$, and $\delta$, and their antibonding counterparts $\left(\sigma^{*}, 2 \pi^{*}\right.$, and $\left.\delta^{*}\right)$. The $\mathrm{Re}_{2}$-core has four eight electrons which are distributed 
among the eight orbitals (Fig. 2) giving a $\operatorname{CAS}(8,8)$ active space. The $\operatorname{CAS}(8,8)$ active space indicates that eight electrons in eight orbitals. The DFT-OLYP geometry is used in the CASSCF calculations with a Re-Re bond length of $2.20 \AA$. . The CASSCF orbitals of the $\left[\mathrm{Re}_{2} \text {-Model }\right]^{0}$ complex are shown in Fig. 3. The ground state wavefunction of the $\left[\mathrm{Re}_{2} \text {-Model }\right]^{0}$ molecule is dominated by $\sigma^{2} \pi^{4} \delta^{2}$ configuration with 0.69 weight of the total wavefunction. The second configuration weights only 0.16 of the total wavefunction is $\sigma^{2} \pi^{4} \delta^{0} \delta^{* 2}$. The rest has a neglectable contribution to the total wavefunction with a contribution less than 0.02. The bond order of the $\sigma$ manifold is 0.93 and for $\pi$ and $\delta$ manifolds are 1.8 and 0.56 , respectively, giving total bond order of 3.29 which is very diverge from the formal bond order of 4.0. This bond order is bigger than the Re-Re bond order of $\left[\mathrm{Re}_{2} \mathrm{Cl}_{8}\right]^{2-}$ predicated by Roos and Sakaki to be 3.20 and 3.18, respectively. ${ }^{10}$

For [Os 2 -Model] ${ }^{0}$ (active space orbitals are shown in Fig. ESI1), the $\sigma^{2} \pi^{4} \delta^{2} \delta^{* 2}$ configuration makes 0.87 of the total wavefunction, the occupations of the $\sigma$ and $\pi$ manifolds are identical to the $\left[\operatorname{Re}_{2}-\right.$ Model $^{0}$ molecule while the occupations of $\delta$ manifold are 2.00 for both $\delta$ and $\delta^{*}$ orbitals. The bond order of the $\delta$ manifold is zero compared to 0.56 of the $\left[\mathrm{Re}_{2}-\mathrm{Model}\right]^{0}$ molecule. The total bond order of $\left[\mathrm{Os}_{2}-\mathrm{Model}^{0}\right.$ molecule is 2.73 which is much smaller than the bond order of the $\left[\operatorname{Re}_{2}-\text { Model] }\right]^{0}$ (3.29). For both $\left[\operatorname{Re}_{2^{-}}\right.$ Model $]^{0}$ and $\left[\mathrm{Os}_{2}-\text { Model }\right]^{0}$ complexes, the active space was expanded to include two $\mathrm{M}-\mathrm{N} \delta(\mathrm{M}=\operatorname{Re}$ or Os) bonding orbitals and the corresponding two antibonding molecular orbitals and them four electrons distributing among them. This yields $\operatorname{CAS}(12,12)$ and $\operatorname{CAS}(14,12)$ active space for $\left[\mathrm{Re}_{2}-\mathrm{Model}^{0}\right.$ and $\left[\mathrm{Os}_{2^{-}}\right.$ Model] $]^{0}$, respectively (see Fig. ESI2 and Fig. ESI3). For [ $\operatorname{Re}_{2}-$ Model] ${ }^{0}$, the $\operatorname{Re}-\mathrm{N} \delta$ orbitals are kept fully occupied with the occupation of 1.99 and 0.01 of bonding and antibonding orbitals, respectively. The occupations of the $\sigma$ and $\pi$ manifolds are the same to that in the $\operatorname{CAS}(8,8)$ whereas the occupations of the $\delta$ manifold is slightly different from those of the $\operatorname{CAS}(8,8)$ with occupations of 1.58 and 0.42 of $\delta$ and $\delta^{*}$ orbitals, respectively, compared to 1.56 and 0.44 in $\operatorname{CAS}(8,8)$. Resulting in slight increase in the strength of the $\delta$ bonding ( $\delta$ bond order of 0.56 with CAS $(8,8)$ compare to 0.58 with CAS $(12,12)$ ) but this has almost no effect on the total bond order (3.30 vs 3.29). For [Os ${ }_{2}-$ Model $]^{0}$, the Os- $N \delta$ orbitals are preserved fully occupied with occupation number of 1.99 and 0.01 of bonding and antibonding orbitals, respectively, similar to those of Re-analogue. The active space orbitals of the $\left[\mathrm{Os}_{2}-\mathrm{Model}\right]^{0}$ are shown in Fig. ESI3. These results show that including the M-N $\delta$ orbitals in the active space has no effect on the nature of the metalmetal bonding in these two complexes. 


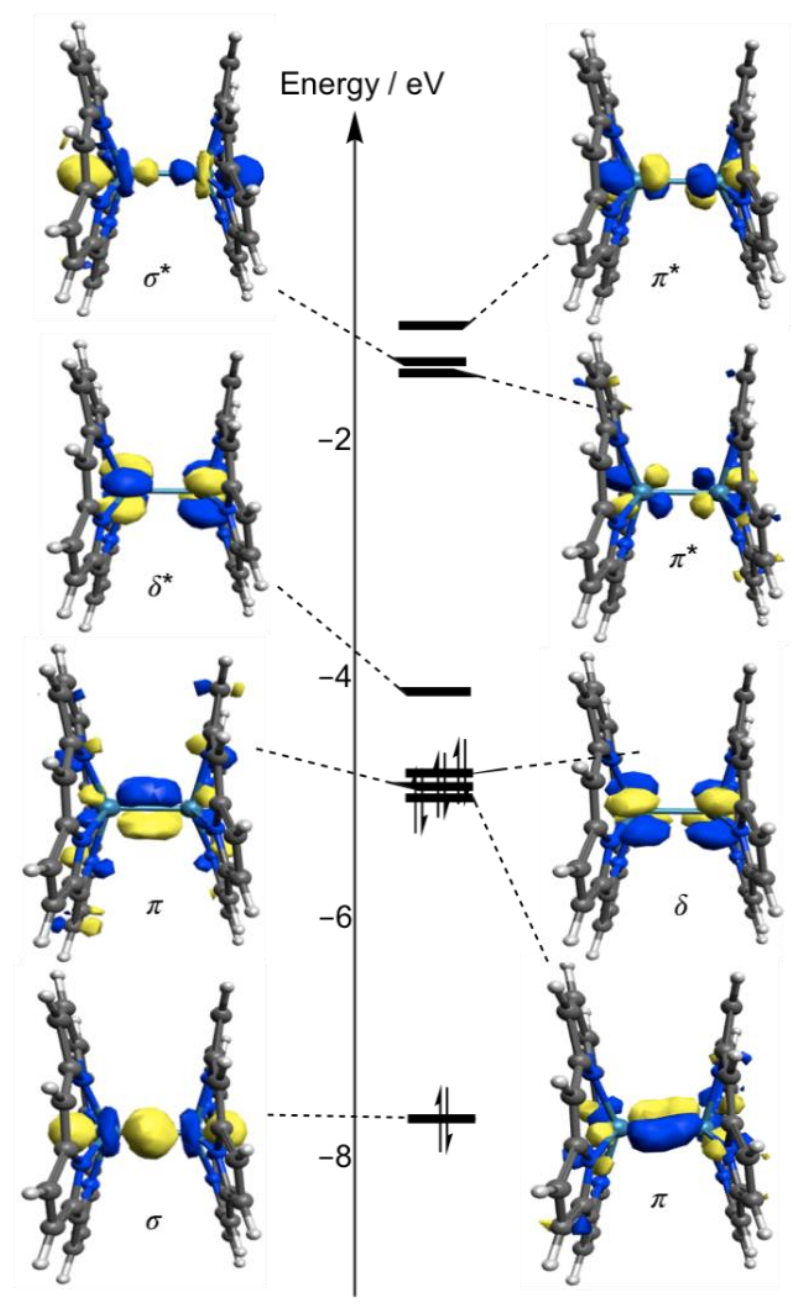

Fig. 2. Kohn-Sham molecular orbital diagram of the $\left[\operatorname{Re}_{2}-M o d e l\right]^{0}$ complex computed using DFT-OLYP.
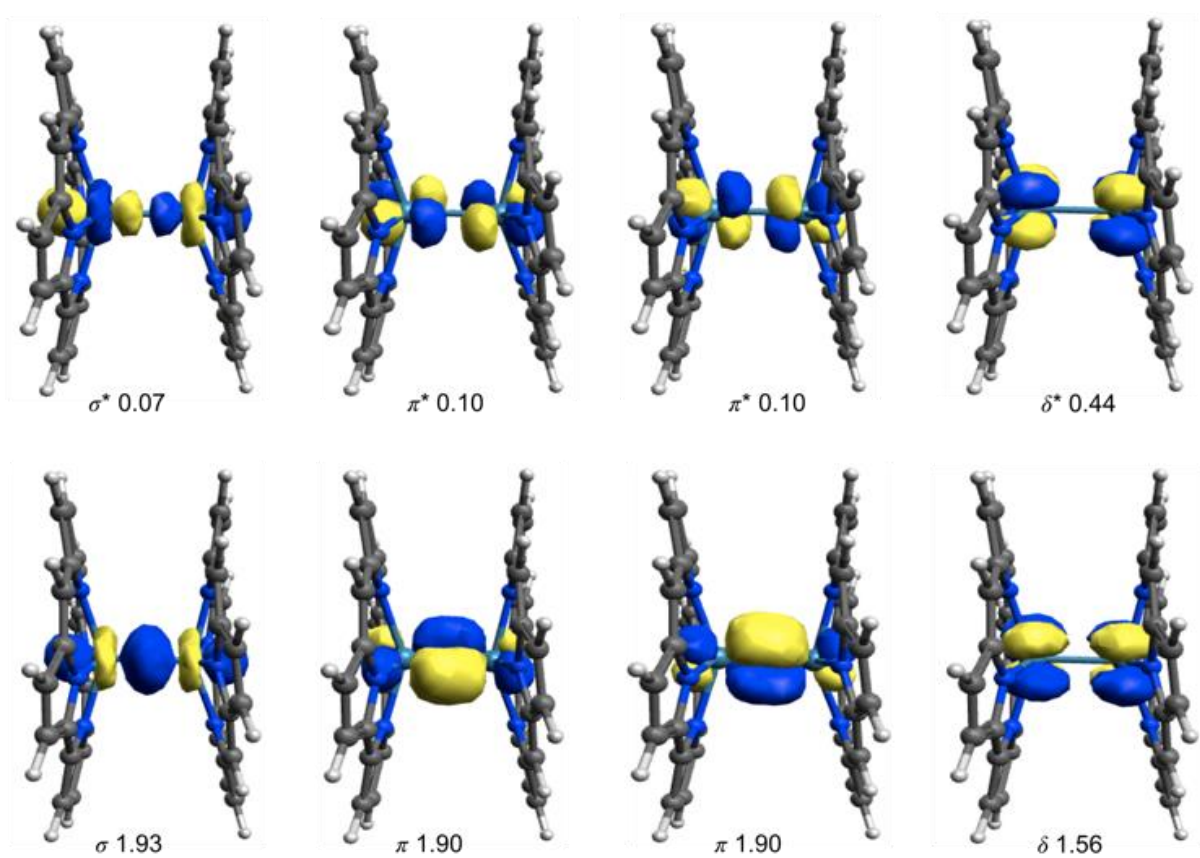

Fig. 3. Active space natural orbitals with occupations of the $\left[\operatorname{Re}_{2}-\operatorname{Model}\right]^{0}$ complex $(\operatorname{CAS}(8,8))$. 
$\left[\mathrm{Re}_{2}-\text { Model }\right]^{1+}$ and $\left[\mathrm{Os}_{2}-\text { Model }\right]^{1+}$ : One electron removal from the $\left[\mathrm{Re}_{2}-\mathrm{Model}\right]^{0}$ system occurs either from the metal-based orbitals or the ligand-based orbitals giving a $\left[\mathrm{Re}_{2}-\text { Model }\right]^{1+}$ molecule. Our DFT using OLYP and B3LYP calculations show that the single electron removal is removed from ligand-based orbitals. Furthermore, the $\delta^{*}$ metal-based orbital is populated as shown in Scheme 1 , one of the $\delta$ manifold electrons is coupled antiferromagnetically to the electron on the ligands giving a ${ }^{2} \mathrm{~A}$ ground state. The Mulliken spin density of 0.98 (OLYP) on each ruthenium centre. The Re-Re bond length of $2.21 \AA$ is slightly longer than that of the neutral molecule $\left[\operatorname{Re}_{2}-\mathrm{Model}\right]^{0}(2.20 \AA)$ suggesting that the populated weakly bonding $\delta$ orbital has a small impact on the total bond strength. This behaviour is also observed using B3LYP, the Mulliken spin density of 1.0 on each metal centre. The Re-Re bond length of $2.24 \AA$ is also slightly longer than in the $\left[\mathrm{Re}_{2}-\text { Model }\right]^{0}$ molecule (2.22 $\AA$ ). Removing an electron for the neutral molecule leads to populating the $\delta^{*}$ orbitals which are consistent with the finding of Ghosh and co-workers. ${ }^{6}$

For $\left[\mathrm{Os}_{2} \text {-Model }\right]^{1+}$ molecule, Mulliken spin density of 0.05 and 0.01 on each Os centre calculated using OLYP and B3LYP, respectively. Those results suggested that the unpaired electron is mainly removed from ligandbased orbitals. However, the Os-Os bond length of $2.21 \AA$ (OLYP) is slightly shorter than in the [Os ${ }_{2}-\mathrm{Model}^{0}$ (2.22 ̊). The spin-density profiles of the [Os 2 -Model] ${ }^{1+}$, shown in Fig. ESI3, indicates that the $\delta^{*}$ (HOMO-2) orbital is fully occupied since if the electron is removed from this orbital, it would then be empty. The removal of an electron from ligand-based orbitals in both $\left[\mathrm{Re}_{2}-\mathrm{Model}\right]^{0}$ and $\left[\mathrm{Os}_{2}-\mathrm{Model}\right]^{0}$ complexes agreeing with the cyclic voltammetry measured by Ghosh and co-workers. ${ }^{6 a, b}$ The first oxidation potentials were found to be nearly identical for both metals ( $0.51 \mathrm{~V}$ for Re and $0.55 \mathrm{~V}$ for Os) suggesting the removal of the electron occurs the same type of orbital. Furthermore, the computed metal-metal bond distances in both systems are slightly changed upon first oxidation compared to neutral molecules confirming the removal is indeed from the ligand-based orbital.

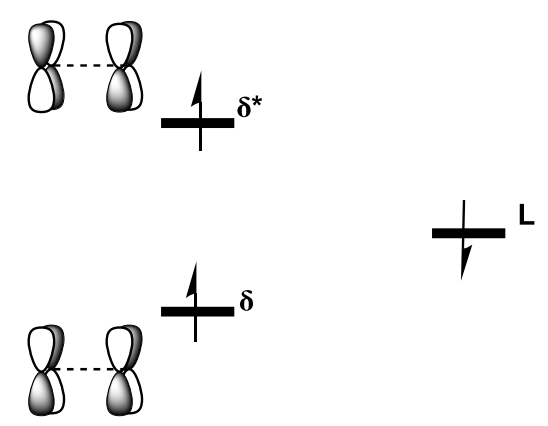

Scheme 1. Systematic representation of the configurations of the $\left[\mathrm{Re}_{2}-\mathrm{Model}\right]^{1+}$ molecule using both OLYP and B3LYP functionals.

To gain insight into the nature of the metal-metal bonding in the $\left[\mathrm{Re}_{2}-\mathrm{Model}\right]^{1+}$ and the $\left[\mathrm{Os}_{2}-\mathrm{Model}^{1+}\right.$ systems, we have run CASSCF calculations using the same active spaces as in their neutral forms. However, 
the geometries of both systems are slightly different from the neutral forms. Furthermore, the $\delta^{*}$ orbital in the $\left[\mathrm{Re}_{2} \text {-Model }\right]^{1+}$ molecule is populated; thus, it needs to consider in the CASSCF calculations. Therefore, $\sigma^{2} \pi^{4} \delta^{2} \delta^{* 0}$ and $\sigma^{2} \pi^{4} \delta^{1} \delta^{* 1}$ will be investigated as they would give different nature of Re-Re bonding. In principle, the $\sigma^{2} \pi^{4} \delta^{2} \delta^{* 0}$ (singlet) configuration gives formal bond order of 4.0 while the $\sigma^{2} \pi^{4} \delta^{1} \delta^{* 1}$ (triplet) configuration gives 3.0. DLPNO-NEVPT2 results show that the triplet state is $0.5 \mathrm{eV}$ higher in energy than the singlet. The active space natural orbitals with occupations of the singlet and the triplet states are shown in Fig. ESI5. The $\sigma^{2} \pi^{4} \delta^{2} \delta^{* 0}$ configuration makes up 0.67 of the total wavefunction, and the second most predominant configuration is $\sigma^{2} \pi^{4} \delta^{0} \delta^{* 2}$ weights only 0.17 of the total wavefunction. The occupations number of the $\sigma$ and $\sigma^{*}$ manifolds are 1.93 and 0.07 , respectively, same as those in the $\left[R_{2_{2}-}\right.$ Model $]^{0}$. The occupations of the $\pi$ and $\delta$ and them antibonding orbitals are slightly different from those of $\left[\mathrm{Re}_{2}-\right.$ Model] ${ }^{0}$ due to different geometries (Re-Re distance of $2.24 \AA$ compared to $2.22 \AA$ of the $\left[\mathrm{Re}_{2}\right.$ Model $\left.]^{\circ}\right)$. The occupations of the $\pi$ and $\pi^{*}$ are 3.78 and 0.22 , respectively. The occupations of the $\delta$ and $\delta^{*}$ are 1.54 and 0.46 , respectively. These occupations give a total bond order of 3.25. The geometry of the $\left[\mathrm{Os}_{2}-\text { Model }\right]^{1+}$ is very similar to the neutral analogue and also the same active space $(\operatorname{CAS}(10,8))$ would be used in the CASSCF calculations (see Fig. ESI1). Therefore, single point CASSCF calculations have the same electrons configuration, the $\sigma^{2} \pi^{4} \delta^{2} \delta^{* 2}$ configuration has also 0.87 weight of the total wavefunction.

$\left[\operatorname{Re}_{2}-\text { Model] }\right]^{1-}$ and $\left[\mathrm{Os}_{2}-\right.$ Model] ${ }^{1-}$ : Adding one electron to the $\left[\operatorname{Re}_{2}-\right.$ Model] ${ }^{0}$ system produces $\left[\operatorname{Re}_{2}-\right.$ Model] ${ }^{1-}$. DFT calculations show that the single electron is added to a metal-based orbital giving Mulliken spin density of 0.52 and 0.50 on each Re centre with OLYP and B3LYP, respectively. The Re-Re bond distance of $2.22 \AA$ (OLYP) in the $\left[\mathrm{Re}_{2}-\mathrm{Model}\right]^{1-}$ is slightly longer than that in the neutral molecule $(2.20 \AA)$ as expected since adding one electron to the weakly overlap $\delta^{*}$ orbital results in decreasing the formal bond order from 4.0 in $\left[\operatorname{Re}_{2}-M o d e l\right]^{0}$ to 3.5 in $\left[\operatorname{Re}_{2}-M o d e l\right]^{1-}$. In contrast to the $\left[\mathrm{Re}_{2}-\mathrm{Model}\right]^{1-}$, the electron is added to the ligandbased orbitals in the $\left[\mathrm{Os}_{2} \text {-Model] }\right]^{1-}$ molecule as suggested by Mulliken density. The Mulliken density on each osmium centre is equal to 0.005 and 0.009 with OLYP and B3LYP, respectively. The Os-Os bond distance predicts to be the same as that in the neutral molecule, [Os ${ }_{2}$-Model] ${ }^{0},(2.22 \AA$ (OLYP)).

The $\left[\mathrm{Re}_{2}-\mathrm{Model}\right]^{1-}$ and $\left[\mathrm{Os}_{2} \text {-Model }\right]^{1-}$ molecules show very different behavior under the reduction potentials, adding an electron to metal-based orbital in the $\left[\mathrm{Re}_{2}-\text { Model }\right]^{1-}$ complex while this electron occupied ligand-based orbital in the $\left[\mathrm{Os}_{2}-\right.$ Model $^{1-}$. These results are in excellent agreement with cyclic voltammetry measurements. The reduction potentials proved to be very different for the two metals $(-0.55 \mathrm{~V}$ for $\mathrm{Re}$ and $-1.31 \mathrm{~V}$ for $\mathrm{Os}) .^{6 a, b}$ The difference in reduction potentials suggests electron addition into a different type of orbitals; this is consistent with our finds.

CASSCF method was applied for further exploring the nature of the metal-metal bonding in the $\left[\mathrm{Re}_{2^{-}}\right.$ Model $]^{1-}$ molecule. A CAS $(9,8)$ active space was adopted, including the eight metal-based orbitals and nine electrons distributed among them using the DFT-OLYP geometry (Re-Re bond distance of $2.22 \AA \AA$ ). The active space natural orbitals with occupations of the $\left[\mathrm{Re}_{2}-\mathrm{Model}\right]^{1-}$ are appeared in Fig. ESI6. The 
$\sigma^{2} \pi^{4} \delta^{2} \delta^{* 1}$ configuration makes up $85 \%$ of the total wavefunction of the ${ }^{2} \mathrm{~A}$ ground state. The occupations of the $\sigma$ and $\sigma^{*}$ orbitals are 1.93 and 0.07 , respectively, giving a bond order of 0.93 . The bond order of the $\pi$ - and $\delta$-mainfold are 1.82 and 0.43 , respectively. Thus, effective bond order in the $\left[\mathrm{Re}_{2}-\mathrm{Model}\right]^{1-}$ molecule is 3.18 compared to 3.29 in the $\left[\mathrm{Re}_{2}-\text { Model }\right]^{0}$ molecule.

\section{Conclusions}

The electronic structure of neutral, oxidized, and reduced species of dinuclear rhenium and osmium corrole complexes, $\left[\{\operatorname{Re}[\mathrm{TpXPC}]\}_{2}\right]^{0 / 1+/ 1-}$ and $\left[\{\mathrm{O}[\mathrm{TpXPC}]\}_{2}\right]^{0 / 1+/ 1-}$, were theoretically investigated using state-of-art quantum methods including density functional theory (DFT) and complete active space self-consistent field (CASSCF) methodologies. The DFT computed ground state geometry of both $\left[\{\operatorname{Re}[\mathrm{TpXPC}]\}_{2}\right]^{0}$ and $\left[\{\mathrm{Os}[\mathrm{TpXPC}]\}_{2}\right]^{0}$ complexes are in excellent agreement with the experimental structures determined by single-crystal X-ray diffraction. However, DFT fails in taking into account the very different contribution of metal-metal components; therefore, for a better description, CASSCF were applied. For the $\left[\left\{\operatorname{Re}[\mathrm{TpXPC}\}_{2}\right]^{0}\right.$ and the $\left[\{\mathrm{Os}[\mathrm{TpXPC}]\}_{2}\right]^{0}$ molecules, CASSCF calculations show that the effective bond order is 3.29 and 2.73, respectively. These bond orders vastly diverg from the formal bond order of 4.0 and 3.0 for the $\left[\{\operatorname{Re}[\mathrm{TpXPC}]\}_{2}\right]^{0}$ and the $\left[\{\mathrm{OS}[\mathrm{TpXPC}]\}_{2}\right]^{0}$, respectively; that would expect from a single-determinant DFT method.

For the oxidized species, the oxidation process removes an electron from the ligand-based orbital in both $\left[\{\operatorname{Re}[\mathrm{TpXPC}]\}_{2}\right]^{1+}$ and $\left[\{\mathrm{OS}[\mathrm{TPXPC}]\}_{2}\right]^{1+}$. The Re-Re bond distance is slightly elongated compared to that in the neutral molecule $\left(2.20 \AA\right.$ vs $2.21 \AA$ ) due to populating the $\delta^{*}$ orbital. The ground state $\sigma^{2} \pi^{4} \delta^{2}$ configuration makes 0.67 of the total wavefunction giving effective bond order of 3.25. For the Osanalogue, the effective bond order is 2.73 same as that in the neutral molecule. On the reduction process, the electron was found occupying the metal-based orbital in the $\left[\{\operatorname{Re}[\mathrm{TpXPC}]\}_{2}\right]^{1-}$ whereas in the ligandbased orbital of the $\left[\{\mathrm{OS}[\mathrm{TPXPC}]\}_{2}\right]^{1-}$. Accordingly, that reduction process does not affect on the nature of the Os-Os bonding in the $\left[\{\mathrm{Os}[\mathrm{TpXPC}]\}_{2}\right]^{1-}$. For $\left[\{\operatorname{Re}[\mathrm{TpXPC}]\}_{2}\right]^{1-}$ complex, the CASSCF predicted the effective bond order is 3.18 compared to 3.29 in the neutral form.

We envision that using high-level computations will provide a further understanding of the metalloporphyrin dimers with multiple metal-metal bonds to predict the physical properties and the reactivities of such metal complexes.

\section{Computational details}


All calculations in this paper were performed using ORCA 5.0 package. ${ }^{12}$ Two functionals were used in this work, the OLYP and B3LYP. ${ }^{13,14}$ The OLYP functional have been extensively used in studying metalloporphyrin-type complexes. ${ }^{6 \mathrm{~d}}$ We have used def2-TZVP basis set to describe the Re and Os while N, $\mathrm{C}$, and $\mathrm{H}$ were described using def2-SVP basis set with def2/J auxiliary basis set. ${ }^{15,16}$ The atom-pairwise dispersion correction with the Becke-Johnson damping scheme (D3BJ) were applied. ${ }^{17,18}$ All structures have been optimized in $\mathrm{CH}_{2} \mathrm{Cl}_{2}$ solvent using SMD solvation module. ${ }^{19}$ Single point gas-phase CASSCF calculations used same basis sets in DFT calculations but with def2-svp/c ${ }^{20}$ and def2/J auxiliary basis set. To speed up the CASSCF calculations we have used RIJCOSX approximation. ${ }^{21}$

\section{Conflicts of interest}

There are no conflicts to declare.

\section{Acknowledgements}

The authors would like to acknowledge the use of the University of Oxford Advanced Research Computing (ARC) facility in carrying out this work.

\section{References}

1 W. Sinha, M. G. Sommer, L. Hettmanczyk, B. Patra, V. Filippou, B. Sarkar and S. Kar, Chem. Eur. J., 2017, 23, 2396-2404.

2 H. L. Buckley, L. K. Rubin, M. Chrominski, B. J. McNicholas, K. H. Y. Tsen, D. T. Gryko and J. Arnold, Inorg. Chem., 2014, 53, 7941-7950. (b) H. L. Zhao, K. Pierloot, E. H. G. Langner, J. C. Swarts, J. Conradie and A. Ghosh, Inorg. Chem., 2012, 51, 4002-4006.

3 H.-Y. Liu, T.-S. Lai, L.-L. Yeung and C. K. Chang, Org. Lett., 2003, 5, 617-620. (b) I. Nigel-Etinger, I. Goldberg and Z. Gross, Inorg. Chem., 2012, 51, 1983-1985.

4 J. P. Collman and H. J. Arnold, Acc. Chem. Res., 1993, 26, 586-592. (b) J. P. Collman, C. E. Barnes, T. J. Collins, P. J. Brothers, J. Gallucci and J. A. Ibers, J. Am. Chem. Soc., 1981, 103, 7030-7032. (c) F. R. Hopf, T. P. Obrien, W. R. Scheidt and D. G. Whitten J. Am. Chem. Soc., 1975, 97, 277-281. (d) J. P. Collman, P. J. Brothers, L. McElweewhite, E. Rose and L. J. Wright, J. Am. Chem. Soc., 1985, 107, 4570-4571. (e) J. P. Collman, H. J. Arnold, J. P. Fitzgerald and K. J. Weissman, J. Am. Chem. Soc., 1993, 115, 9309-9310.

5 K. M. Kadish, F. Burdet, F. Jerome, J. M. Barbe, Z. P. Ou, J. G. Shao and R. Guilard, J. Organomet. Chem., 2002, 652, 69-76.

6 (a) B. Alemayehu, L. J. McCormick-McPherson, J. Conradie and A. Ghosh, Inorg. Chem., 2021, 60, 83158321. (b) A. B. Alemayehu, L. J. McCormick, H. Vazquez-Lima and A. Ghosh, Inorg. Chem., 2019, 58, 27982806. (c) A. Ghosh, Chem. Rev. 2017, 117, 3798-3881. (d) S. Nardis, F. Mandoj, M. Stefanelli, and R. 
Paolesse, Coord. Chem. Rev. 2019, 388, 360-405. (e) A. B. Alemayehu, H. Vazquez-Lima, K. J. Gagnon, and A. Ghosh, Inorg. Chem. 2017, 56, 5285-5294.

7 J.E. McGrady, Introduction and General Survey of Metal-Metal Bonds. In Molecular Metal-Metal Bonds, S.T. Liddle (Ed.), 2015, pp. 1-22.

8 (a) F. A. Cotton. F. Curtisc. B. Harrisb. F. G. Johnsons. J. Lippardj. T. Maguew. R. Robinsonand j. S. Wood, Science, 1964, 145, 1305-1307. (b) F. A. Cotton, Inorg. Chem., 1965, 4, 334-336.

9 (a) J. V. Brencic and F. A. Cotton, Inorg. Chem., 1969, 8, 7-10. (b) D. Lawton and R. Mason, J. Am. Chem. Soc., $1965,87,921-922$.

10 (a) L. Gagliardi and B. O. Roos, Inorg. Chem., 2003, 42, 1599-1603. (b) K. Saito, Y. Nakao, H. Sato and S. Sakaki, J. Phys. Chem. A, 2006, 110, 9710-9717.

11 F. Poineau, P. M. Forster, T. K. Todorova, E. V. Johnstone, W. M. Kerlin, L. Gagliardi, K. R. Czerwinski and A. P. Sattelberger, Eur. J. Inorg. Chem., 2014, 4484-4495.

12 F. Neese, F. Wennmohs, U. Becker, and C. Riplinger, J. Chem. Phys., 2020, 152, 224108.

13 C. Lee, W. Yang, and R. G. Parr, Phys. Rev. B: Condens. Matter Mater. Phys., 1988, 37, 785-789.

14 (a) A. D. Becke, J. Chem. Phys. 1993, 98, 5648 - 5652. (b) P. J. Stephens, F. J. Devlin, C. F. Chabalowski, M. J. Frisch, J. Phys. Chem. 1994, 98, 11623 - 11627.

15 F. Weigend and R. Ahlrichs, Phys. Chem. Chem. Phys., 2005, 7, 3297-3305.

16 F. Weigend, Phys. Chem. Chem. Phys., 2006, 8, 1057-1065.

17 S. Grimme, S. Ehrlich, L. Goerigk, J. Comput. Chem., 2011, 32, 1456-1465.

18 S. Grimme, J. Antony, S. Ehrlich and H. Krieg, J. Chem. Phys., 2010, 132, 154104.

19 A. V. Marenich, C. J. Cramer and D. G. Truhlar, J. Phys. Chem. B, 2009, 113, 6378-6396.

20 A. Hellweg, C. Hättig, S. Höfener and W. Klopper, Theor. Chem. Acc., 2007, 117, 587-597.

21 C. Kollmar, K. Sivalingam, B. Helmich-Paris, C. Angeli and F. Neese, J. Comput. Chem., 2019, 40, 1463-1470. 